Musées, Patrimoine et Culture scientifiques et techniques

149 | 2013

septembre-octobre 2013

\title{
Des seniors au muséum : une nébuleuse de projets
}

\section{Caroline Grandjean et Marlène Prost}

\section{OpenEdition \\ Journals}

Édition électronique

URL : https://journals.openedition.org/ocim/1282

DOI : $10.4000 /$ ocim. 1282

ISSN : 2108-646X

Éditeur

OCIM

Édition imprimée

Date de publication : 27 septembre 2013

ISSN : 0994-1908

Référence électronique

Caroline Grandjean et Marlène Prost, «Des seniors au muséum : une nébuleuse de projets », La Lettre de l'OCIM [En ligne], 149 | 2013, mis en ligne le 27 septembre 2015, consulté le 21 septembre 2021. URL : http://journals.openedition.org/ocim/1282 ; DOI : https://doi.org/10.4000/ocim.1282 
mémoire, l'attention, la compréhension et le raisonnement.Certainespeuventnotammentdévelopperdes pathologies démentielles comme les maladies d'Alzheimer ou de Parkinson. À noter que le terme « démence » au sens médical fait uniquement référence à ces maladies entraînant des troubles cognitifs et noncognitifs, il n'est donc pas question de folie comme le laisse supposer l'utilisation de ce mot dans le langage courant. La maladie d'Alzheimer se divise en quatre phases : la première est caractérisée par des troubles de la mémoire immédiate et la deuxième par des troubles du langage du geste, de la reconnaissance et de l'attention. Dès la troisième phase, l'autonomie des patients est très fortement diminuée. Il leur est alors impossibledesedéplacerjusqu'aumuséum-aquariumpour suivre une visite commentée. Tout comme pour celle d'Alzheimer, la maladie de Parkinson provoque une dégénérescenceintellectuelleàlaquellesecouplentun ralentissement moteur et une plus grande difficulté dans l'accomplissement des gestes du quotidien.

À ces troubles cognitifs plus ou moins avancés, peuvent s'ajouter une fatigue cardiaque ou respiratoire, des problèmes d'orientation, des difficultés de déplacement induisant l'utilisation de cannes, déambulateurs ou fauteuils roulants, ou encore des troubles de la vision ou de l'audition.

\section{La genèse du projet}

Début 2011, des visites commentées sont proposéesà depetitsgroupesdepersonnesâgéesatteintes desmaladies d'Alzheimer ou de Parkinson (groupe de trois personnes maximum). Elles remportent un franc succès. Elles sont à l'initiative d'une ergothérapeute qui les intègre à ses visites hebdomadaires à domicile. II s'agit donc là de personnes encore autonomes, dans les $1^{1 \text { èr }}$ ou $2^{\mathrm{e}}$ phases de la maladie.

Les activités sont conçues par les médiatrices du muséum-aquarium,enconcertationaveccetteprofessionnelle. Les thématiques traitées portent sur les animaux de la ferme et les animaux de Lorraine. Plusieurs spécimens issus des collections du muséum-aquarium permettent d'illustrer le propos. La proximité avec les collections permet d'ouvrirunvéritabledialogueavecdes personnes qui, au quotidien, ont parfois de grandes difficultés à verbaliser. Leur mémoire est plus facilement sollicitée, ce qui les incite à prendre la parole pour enrichir la visite.

Stimulés par cette première expérience réussie, une idéeémergealors: proposer un véritable programme de visites à l'attention de personnes atteintes de ces maladies en s'appuyant sur la diversitédes spécimens descollections, patrimonialesetpédagogiques.Pource faire, une note est adressée au président du Grand Nancy afin qu'il valide le projet et facilite le rapprochement avec les spécialistes de la gérontologie sur le territoire.
Elle est accueillie très favorablement et les perspectives d'échangeaveclesautres musées del'agglomération nancéienne sont immédiatement soulignées.

\section{Le projet multi-partenarial \\ « des seniors au muséum »}

\section{Sur le territoire du Grand Nancy}

Le muséum-aquarium propose au CCAS (2) de Nancy et au service de cohésion sociale du Grand Nancy de s'associer au projet. L'objectif est d'une part, de profiter de leurs connaissances de ce public spécifique et du réseau des structures gérontologiques sur le territoire et d'autre part, de bénéficier de relais pour communiquerl'information. Dans un premier temps, on s'oriente vers les maisons de retraite.

Un calendrier de travail est établi. En premier lieu, lesmédiatrices du muséum-aquarium rencontrentdes

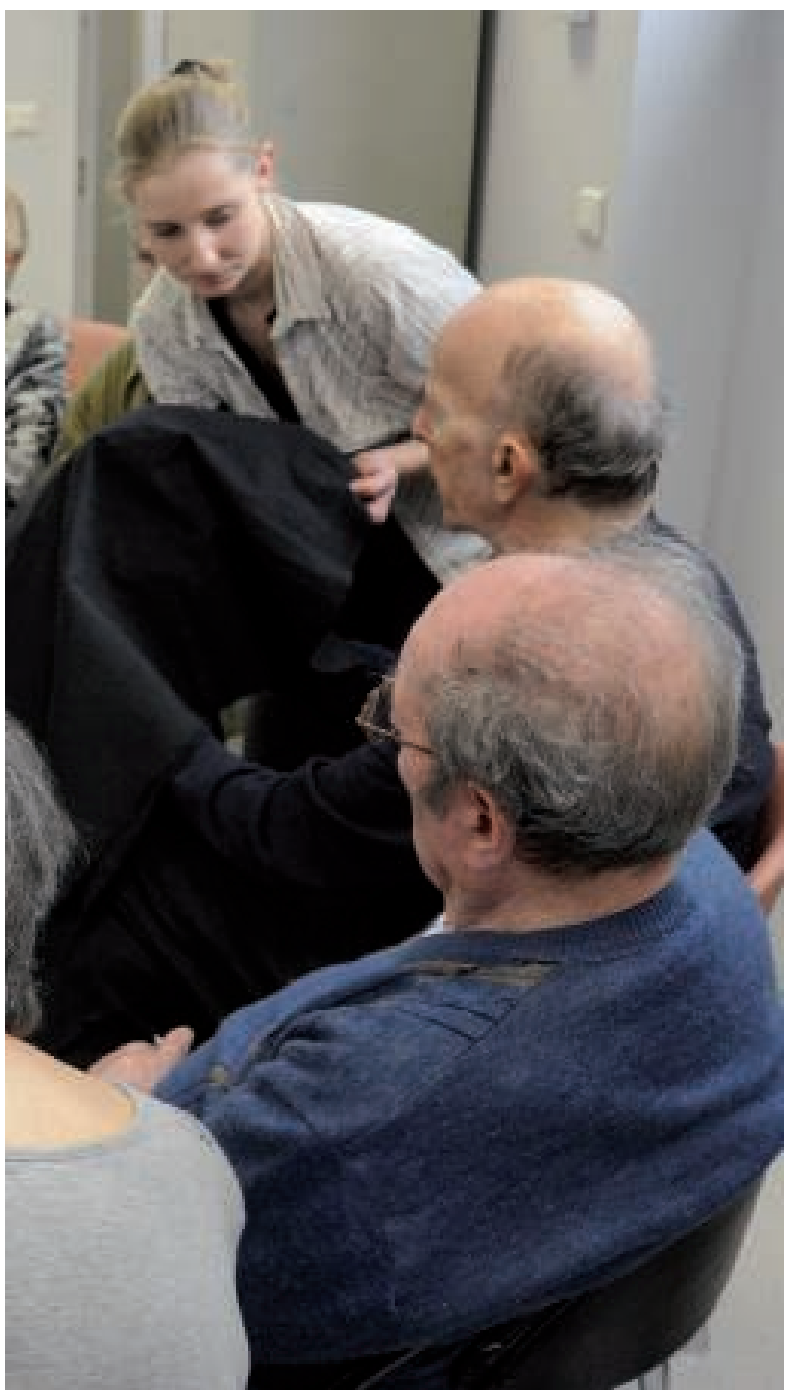

Toucher un animal à l'aveugle pour deviner de quoi il s'agit. (c) Muséum-aquarium de Nancy 
professionnels deladisciplineafin demieuxconnaître le cœur de cible et ainsi mieux répondre à ses particularités. Cet échange se fait avec des personnels de I'EHPAD (3) "Notre Maison » de Nancy : une géronto-psychologue et une animatrice.

Dès la présentation du projet, une incohérenceest mise en lumière: proposer des visites strictement réservées à des personnes atteintes des maladies d'Alzheimer ou de Parkinson rentre en contradiction avec l'obligation de conserver le secret médical.

Le projet «Seniors au Muséum » se réoriente alors plusgénéralementversl'ensembledespersonnesâgées résidant en maison de retraite. Par leur connaissance fine du public, une visite des locaux et le suivi d'une activité à destination des résidents, les professionnelles de l'EHPAD permettent aux médiatrices de mieux cerner les particularités de l'auditoire : les différences entre les seniors deviennent une force et servent de moteur pour mener une activité.

À l'issue de cette première étape, une visite thématiquedumuséum-aquariumestconçue,ens'appuyant sur les enseignements récoltés à « Notre Maison » et les remarques des deux spécialistes. Elle est ensuite testée grandeur nature avec un groupe de résidents. Après un bilan avec les accompagnatrices, un retour positif est fait auprès du CCAS et du service de cohésion sociale. Il leur est alors proposé de passer à l'étape suivante:communiquer l'opération dans le réseau. Précisément, il s'agit du réseau gérontologique Gérard Cuny qui couvre le territoire du Grand Nancy ${ }^{(4)}$. Le but est de présenter la démarche du muséum-aquarium et les visites proposées lors d'une « commission seniors » qui réunit tous les acteurs de la gérontologie du Grand Nancy : les EHPAD et les associations, ceci sous la direction de la conseillère communautaire déléguée au handicap et du président de l'Office Nancéien des Personnes Agées ${ }^{(5)}$.

Aucune « commission seniors » n'ayant pu être organisée à cette époque, le CCAS nous a proposé de communiquer sur notre projet via une réunion regroupant les directeurs de foyers-résidence de la Ville de Nancy ${ }^{(6)}$. II en ressort que les structures sont en demande de ce type de prestations mais ne savent pas toujours auprès de qui s'adresser. Au-delà de l'intérêt immédiat de diffusion de l'information, c'est à moyen terme que les résultats de cette rencontre devraient se faire sentir, au travers de la réservation de visites commentées au muséum-aquarium.

\section{Le jardin pour des seniors}

atteints de la maladie d'Alzheimer

Le CHU de Nancy dispose d'une unité Alzheimer au sein de laquelle un jardin des 5 sens a été construit. Ce jardin, intitulé « Art, mémoire et vie », est librement

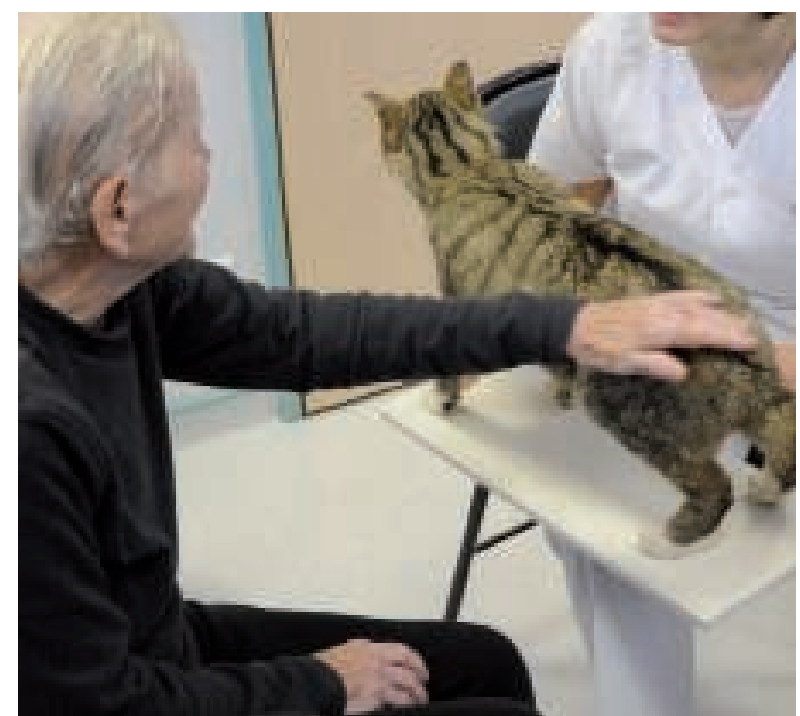

Animation au $\mathrm{CHU}$ avec des spécimens de la collection de prêt et de pédagogie. ๑) Muséum-aquarium de Nancy

accessibleauxpersonnesâgées démentesetestrégulièrement le support d'activités faisant intervenir des structures culturelles locales. Il est composé de quatre carrés, représentant les quatre éléments (eau, terre, feu, air). Dans le cadre de la démarche menée au muséum-aquarium, il semblait indispensable de rencontrer la neuropsychologue à l'initiative de la mise en place de ce jardin. L'objectif était de visiter le lieu, d'exposer notre démarche à cette professionnelle et d'évoquerdespistesd'animationscommunespossibles. Dès la première rencontre, les ressources du jardin et les visites commentées sont apparues complémentaires : les médiatrices décident donc de se déplacer pour aller directement à la rencontre des patients du $\mathrm{CHU}$, dans l'incapacité de se rendre au muséumaquarium. En effet, la thématique « Animaux de la mare » peut très facilement s'intégrer au carré « eau » dujardin thérapeutique:les spécimens et documents pédagogiques viennent parfaitement compléter ce qui peut déjà être observé in situ.

Au final, la collaboration avec l'unité de soin se traduit par la participation à une étude devant aboutir à unepublication.Laneuropsychologueresponsablesouhaite étudier les réactions des patients sur le long terme afin d'évaluer l'influence que peut avoir l'animation sur leur comportement. Trois animations thématiques ont déjà été conçues et testées par le muséum-aquariumetpeuventd'oresetdéjàservirdebase de travail. L'organisation est la suivante : une animation est programmée tous les deux mois (février, avril et juin 2013). Chacune est intégralement filmée afin que l'équipe médicale puisse ensuite analyser les postures, les prises de paroles et les différentes réactions des patients. Pour des raisons climatiques, les 
deux premières animations sont prévues pour être réalisées à l'intérieur de l'hôpital, dans une salle d'animation. Chaque groupe est constitué de huit patients maximum, encadrés par une médiatrice du muséumaquarium et deux personnels soignants.

Une tentative de visite commentée

De réunion en réunion, de nombreux acteurs de la gérontologieen Lorraineontétérencontrés, tous plus intéressés les uns que les autres par les propositions du muséum-aquarium. C'est notamment le cas de I'EHPAD « Saint-Charles » de Bayon (54). Dans cet établissement se trouve une unité Alzheimer au sein de laquelle les seniors sont plus atteints par la maladie que ceux que nous avons rencontrés jusqu'alors. Fin 2012, la neuropsychologue de la structure prend contactavec le muséum-aquarium pour discuter précisément des activités possibles. Bien que les propositions soient tout à fait en phase avec ce qu'elle recherche pour ses résidents, elle n'est pas en mesure de régler le forfait « visite guidée » de $50 €$ (pour les 3 résidents qu'elle peut amener). L'alternative est alors une visite en autonomie, gratuite, avec la mise à disposition de matériel pédagogique.

Depuis mai 2013, les tarifs ont été adaptés afin de pouvoir intégrer ce type de prestation encadrée par un médiateur et imposant un nombre très limité de visiteurs. Désormais, la réservation d'une visite commentée pour 15 personnes maximum est facturée 26 e.

\section{Un public, des collections et des visites}

\section{L'accueil du public senior}

Bien que très hétérogène, le public senior peut être mis à l'aise et en confiance au sein de l'institution muséale en suivant certaines règles simples et élémentaires. Elles ont principalement trait à la manière de s'adresser aux personnes : parler fort, les regarder lorsqu'on leur parle, faire des phrases avec des mots simples, se montrer souriant et attentionné. La construction de l'activité est également primordiale. II s'agit d'aménager des moments ludiques stimulantle maximum de sens chez les personnes du groupe, de prévoir des déplacements réduits au sein de l'établissement et de favoriser la position assise. Dans la pratique, cela induit que la visite soit conçue pour être suivie assis avec un ou deux déplacements seulement et qu'elle se déroule sans perturbation sonore extérieure : l'accueil des autres groupes est décalé à un horaire ultérieur.

En amont d'une animation, un échange est indispensableavecl'équiped'accompagnantsafindemieuxconnaîtrelesindividualitéscomposantlegroupe(nombre de personnes en fauteuil roulant, non voyantes ou démentes).
Les collections du muséum-aquarium... La mémoire sensitive étant l'une de celles qui reste le plus longtemps, c'est sur les cinq sens qu'une grande part du discours s'appuie. Pour ce faire, la collection patrimoniale, présentée defaçon permanentedansla galerie dezoologie, et la collection de prêtet de pédagogie, sans valeur patrimoniale et utilisée uniquement pendant les médiations, jouent un rôle complémentaire. L'immersion dans la thématique de la visite se fait par l'observation de la première et l'analyse d'un détail (scruter la forme d'une patte, caresser un plumage) passe par la seconde. Ce fonctionnement s'explique par trois raisons. D'une part, les collections patrimoniales sontengrande partie présentées sous vitrine, leur accès n'est donc pas aisé (un spécimen peut être présenté en hauteur tandis que la contemplation d'un autre dans sa totalité nécessite de faire le tour complet d'une vitrine). Ensuite, comme nous l'avons déjà souligné, les seniors peuvent avoir des troubles de la vision plus ou moins aigus qui nécessitent la proximité avec l'objet dès lors que l'on souhaite discuter du détail de l'anatomie d'un animal. Enfin, s'agissant de collections patrimoniales, au-delà

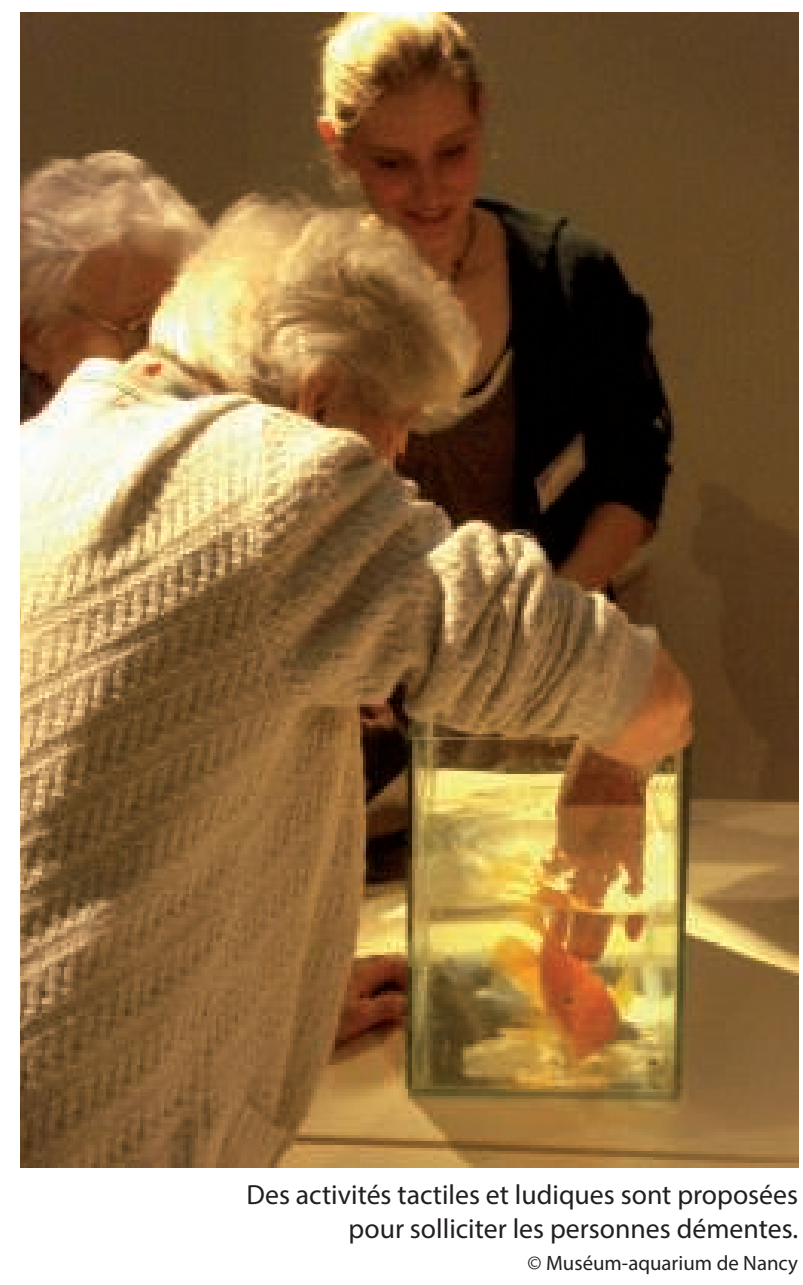


de leur valeur, elles peuvent contenir des produits chimiques toxiques qui les rendent impropres à une manipulation sans protection (gants, masque, blouse). Les spécimens de la collection de prêt et de pédagogie viennent pallier ces difficultés. Naturalisés récemment avec des produits compatibles avec ces pratiques, ils peuventêtremanipulésetobservésminutieusement.II peut s'agir d'un dindon ou d'un agneau naturalisé, du squelette d'une grenouille, d'un test d'oursin ou d'un modèle d'escargot.

\section{... et les activités qui en découlent}

Comme pour n'importe quelle activité de médiation, il s'agit de s'appuyer sur les connaissances de tout ou partie de l'auditoire pour l'amener à en découvrir de nouvelles, ceci en veillant à entretenir les échanges à l'intérieur du groupe tout au long du parcours. Ce souci du dialogue entre participants (médiateur inclus) est d'autant plus primordial qu'il peut permettre ici à certains individus plutôt réservés de s'exprimer. Comme nous l'avons déjà dit, le groupe de seniors est constitué de personnes avec des déficiences et des capacités intellectuelles inégales. Bien queles animations pédagogiques proposées parlemuséum-aquarium n'aient pas de vocation thérapeutique, il est important de prendre en considération le fait que les professionnelsdelagérontologies'ensaisissentgénéralement par la suite pour nourrir leur travail. En effet, de la même manière que les objectifs des animations proposées aux scolaires sont définis en prenant en compte le programme de chaque niveau de classe, il est important de répondre aux attentes des responsables de maisons de retraite.

Afin de ne mettre en difficulté aucun des participants, le dialogue peut s'amorcer de plusieurs façons : suite à la manipulation d'un objet, à l'écoute d'un son, à l'observation d'un animal naturalisé ou à une série de questions. La multiplication des media utilisés permetà chacun de s'exprimer selon ses compétences et son ressenti. Ainsi, celui qui est resté muet pendant toute la visite peut commencer à s'exprimer à partir du simple fait de caresser un lapin et finir par nommer toutes les races qu'il connaît. Un autre ose participer dès lors que tout le groupe doitsentirlecontenu d'uneboîteetproposerdenommercequ'ellecontient. Ces groupes se différencient finalement assezpeu d'autres comme ceux constitués de très jeunes enfants, d'adultes handicapés mentaux ou d'élèves d'ITEP (7). Ils sont relativement réduits en nombre (de 3 à 15 membres). L'interactivité et la manipulation d'objets occupent une place importante et facilitent la participation. Ici, des personnes incapables de dire un mot habituellement vont parfois s'exprimer (à la surprise des accompagnateurs). Le rôle du médiateur est primordial. II rythme la visite, donne la parole équitablement et sait rebondir sur les remarques ou attitudes

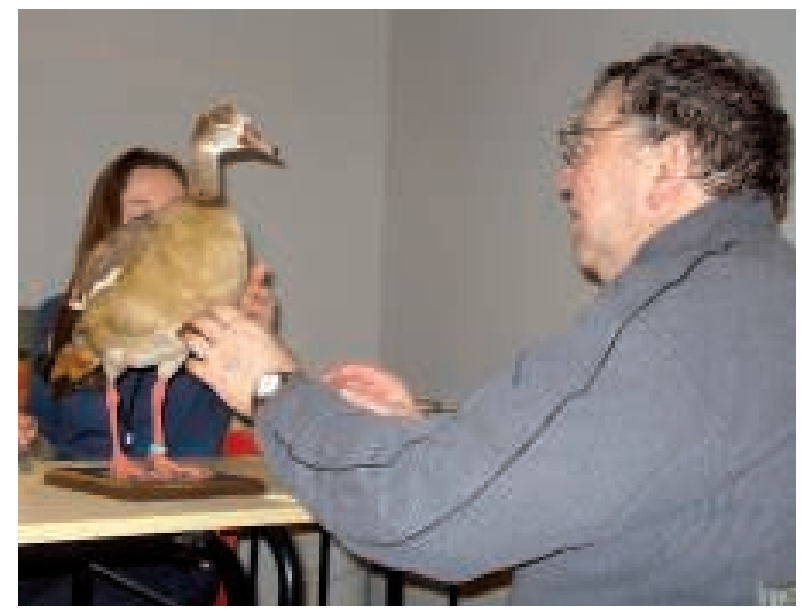

L'observation et la manipulation des spécimens entraînent souvent le récit d'expériences vécues. ○ Muséum-aquarium de Nancy

de chacun. Il doit surtout s'adapter constamment aux réactions de ses interlocuteurs et donner la parole à celui qui jusqu'alors était resté en retrait. Il veille à ce que chacun se sente impliqué dans l'activité.

Unevisitenécessitela mobilisation de plusieurs agents du muséum-aquarium : pour animer et pour faire passer les objets à manipuler (deux médiateurs) mais également pour s'occuper des chaises lors des déplacements (une personne). Le seuil de quinze participants est maximal pour assurer un bon déroulement et éviter les temps morts lorsque chacun doit toucher le même objet par exemple.

La visite «Animaux des 5 continents », par exemple, permetd'aborderlagaleriedezoologiedumuséumaquarium sous un anglegéographique plutôt que biologique. Prenant comme point de départ un planisphère de grande taille, elle est l'occasion de présenter un animal emblématique de chacun des cinq continents. Pour ce faire, cinq activités différentes sont proposées, chacunefaisantappelàdiversescompétences. Le public est invité à toucher en aveugle un spécimen à poils et deviner son nom (un lapin), il doit observer et toucher des éléments liés à un animal à découvrir (un œuf et des plumes d'autruche), il doit deviner un animal à partir d'informations brèves transmises à la façon du jeu «Questions pour un champion!» ou encore, il doit retrouver un spécimen exposé à partir d'une photographieprésentantundétaildesoncorps. Toutes ces activités sont l'occasion de discussions vives et de récits personnels de la part du public.

\section{Conclusion}

Au-delàdelaconception denouvellesanimations, cette démarche "Seniors au Muséum » induit une réelle dynamique de travail. 
Elle conduit à réfléchir à un nouveau public pour le muséum-aquarium : les seniors résidant en maisons de retraite. Cela nécessite un important travail en amont pour arriver à avoir une connaissance fine de ces nouveaux interlocuteurs et leur proposer des animations adaptées.

Dans ce cadre, les multiples rencontres avec des professionnels de la gérontologie nourrissent constamment la réflexion et conduisent à envisager de nouvelles orientations de travail. Il est certain que lors de la conception de la première animation, les médiatrices du muséum-aquarium étaient loin d'imaginer la richesse qui en découlerait (partenariat, échanges, thématiques nouvelles...).

Ce public étant très hétérogène et les animations imposant des conditions particulières (très petits groupes, aucuneperturbationextérieure,nombreuxpersonnels forméspourencadrercesgroupes...),cettedémarche nécessite d'adapter le fonctionnement même de l'accueil du public au sein de l'établissement (changement de la grille tarifaire, du planning de réservations...). Enfin, en accord avec la présidence du Grand Nancy et dans le but d'apporter une dimension territoriale au projet, il est maintenant question de développer un partenariat étroit avec les autres structures muséales de l'agglomération nancéienne.

\section{Notes}

(1) Projet Meet me, pour rendre l'art accessible aux personnes démentes: www.moma.org/meetme/

(2) CCAS : Centre Communal d'Actions Sociales

(3) EHPAD : Établissement d'Hébergement pour Personnes Âgées Dépendantes

(4) Le rôle du réseau est d'assurer et d'organiser l'accès à des soins et des aides en préservant chaque fois que possible le désir de la personne de resteràsondomicile:www.geronto-sud-lorraine.com/le-reseau-gerard-cuny (5) Depuis 1971, I'Office Nancéien des Personnes Âgées prend en charge I'animation, la coordination et la promotion des actions en faveur des seniors : www.onpa.fr

(6) Répartis dans sept quartiers différents, ils accueillent des personnes âgées autonomes dans des appartements qui leur assurent une indépendance de vie équivalente à celle d'un domicile personnel, tout en offrant une sécurité à tout moment, de jour comme de nuit. De nombreuses activités sont proposées tout au long de l'année.

(7) Institut Thérapeutique, Éducatif et Pédagogique.

\section{Bibliographie}

Barroche, G. Les troubles de la mémoire : le point de vue du neurologue, Univers-cité, la revue culture et science de l'université de Lorraine, $n^{\circ} 4$, pp. 40-43.

Bourges, J.-L. Musées et seniors : chronique d'une rencontre annoncée, La Lettre de I'OCIM, n¹33, janvier-février 2011, pp. 24-30. 